\title{
Fuzzy Representation and Aggregation of Fuzzy Cognitive Maps
}

\author{
Mamoon Obiedat and Sandhya Samarasinghe \\ Integrated Systems Modelling Group, Centre for Advanced Computational Solutions (C-fACS), \\ Lincoln University, Christchurch, New Zealand. \\ Email: mamoon.obiedat@lincolnuni.ac.nz
}

\begin{abstract}
Typically, complex systems such as socio-ecological systems are ambiguous and ill-defined due to human-environment interactions. These systems could be participatory systems which involve many participants with different levels of knowledge and experience. The various perceptions of the participants may need to be combined to get a comprehensive understanding and useful knowledge of the system. Modelling these systems involves a high level of uncertainty and soft computing approaches based on the concept of fuzzy logic offer a way to deal with such uncertainty. Fuzzy cognitive map (FCM) incorporates fuzzy logic and has proven its efficiency in modelling and extracting knowledge from various qualitative complex systems. However, the literature shows a lack of appropriate ways to incorporate imprecise human perception in fuzzy form in FCM representation and to deal with these fuzzy values in aggregation of multiple FCMs into a group FCM. The aim of this paper is to provide adequate methods for both representation and aggregation of fuzzy values in FCMs. For FCM representaion, this paper utilizes a 2-tuple fuzzy linguistic representation model Herrera and Martinez (2000a) to represent the FCM connection values in a fuzzy way. This model can represent and deal with linguistic and numeric fuzzy values without any loss of information, and it keeps the consistency of these values throughout any subsequent computational processes.
\end{abstract}

For FCM aggregation, which is the first step, this paper proposes a fuzzy method to combine linguistic and numeric fuzzy values at the same time. In the second step, it proposes a new calculation method to assess the different levels of knowledge of FCM designers (FCMs' credibility weights). These credibility weights of FCMs are then used in the proposed fuzzy aggregation method for a better representation of contrasts between participants resulting from their varied experiences and preferences. For the first step, the 2-tuple fuzzy model is used to represent the FCM connection values during the aggregation process, and therefore the connection values of the group FCM resulting from the aggregation process will be fuzzy values. For the second step, this paper utilizes the Consensus Centrality Measure (CCM) proposed in Obiedat et al. (2011) to calculate a credibility weight for each FCM.

Keywords: Fuzzy cognitive map, 2-tuple fuzzy linguistic representation model, consensus centrality measure, credibility weight, FCM fuzzy representation, FCM fuzzy aggregation 


\section{INTRODUCTION}

FCM is a soft computing approach introduced in 1986 by Kosko (1986). It can represent the knowledge about various complex problems and solve them in the form of fuzzy nodes and connections between nodes. Figure 1, an example of FCM, depicts the causal knowledge of a water scarcity problem. The connections are directed, signed, and carry weighted values. The direction of the connection is to show which node influences which node while its sign indicates the type of influence either a direct (positive) or inverse (negative) influence. The weight value indicates the strength of the influence and it is expressed by numeric fuzzy values in the interval $[-1,1]$ or linguistic values such as low, medium and high etc. From Figure 1, we can see that the 'water scarcity' is negatively and positively influenced by 'Water Resources' and 'Water Demand' respectively. It means that the abundance of 'Water Resources' leads to diminishing 'Water Scarcity', while increasing 'Water Demand' intensifies 'Water Scarcity'. The fuzzy values give the FCM a significant ability to deal with imprecise and uncertain data. Moreover, these fuzzy values provide the experts or developers of FCMs with much freedom and comfort in the assignment of connections between the nodes.

FCM plays a significant role in helping the decisionmaker in modelling the complex dynamics of the system, particularly if the system consists of different participants and stakeholders (Dickerson \& Kosko, 1994; Ozesmi \& Ozesmi, 2003, 2004; Strickert et al., 2010).

Once the FCM is built, it is easily coded into an adjacency matrix. Table 1 shows the adjacency matrix of the FCM in Figure 1. Each element in the matrix

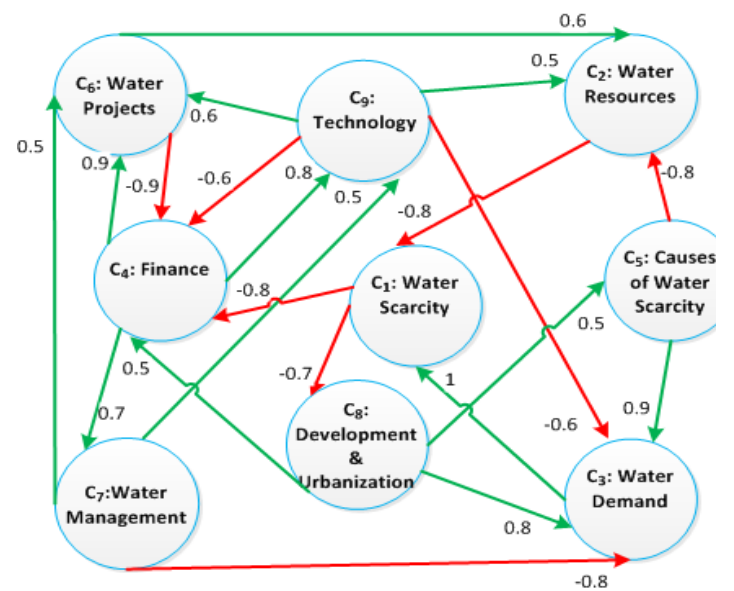

Figure 1. An example FCM depicting the perception of a stakeholder on the Water Scarcity Problem (red- negative, green-positive, and numbers indicate the strength of influence. describes a connection between two nodes. Once the matrix is created, any other calculation processes could be easily performed. One of the important FCM processes is the aggregation of many FCMs into one FCM called group or social FCM. This group or social FCM should represent an overall perception of the problem. The aggregation process is considered a very significant process in the participatory problems that include different perceptions of various participants/stakeholders; many real-life problems fall into this category.

Most real-life problems require the integration of stakeholder perceptions to obtain sufficient and efficient solutions. As the extent of the knowledge and expectations of stakeholders who produce their FCMs vary, it is inevitable that conflicts and issues of credibility arise from such diverse perceptions. In addition, such problems are typically characterised by ambiguity and uncertainty, and therefore different people may use linguistic terms or numeric values to express their knowledge in the form of nodes and connections between nodes. Indeed, such issues constitute a barrier to reaching a valid and accurate group FCM/perception, and therefore, efficient FCM representation and aggregation methods should overcome these issues in order to achieve a consensus perception in a robust and accurate manner.

Table 1. The adjacency matrix of the FCM in Figure 1.

\begin{tabular}{|l|l|l|l|l|l|l|l|l|l|}
\hline & $\mathrm{C}_{1}$ & $\mathrm{C}_{2}$ & $\mathrm{C}_{3}$ & $\mathrm{C}_{4}$ & $\mathrm{C}_{5}$ & $\mathrm{C}_{6}$ & $\mathrm{C}_{7}$ & $\mathrm{C}_{8}$ & $\mathrm{C}_{9}$ \\
\hline $\mathrm{C}_{1}$ & 0 & 0 & 0 & -0.8 & 0 & 0 & 0 & -0.7 & 0 \\
\hline $\mathrm{C}_{2}$ & -0.8 & 0 & 0 & 0 & 0 & 0 & 0 & 0 & 0 \\
\hline $\mathrm{C}_{3}$ & 1 & 0 & 0 & 0 & 0 & 0 & 0 & 0 & 0 \\
\hline $\mathrm{C}_{4}$ & 0 & 0 & 0 & 0 & 0 & 0.9 & 0.7 & 0 & 0.8 \\
\hline $\mathrm{C}_{5}$ & 0 & -0.8 & 0.9 & 0 & 0 & 0 & 0 & 0 & 0 \\
\hline $\mathrm{C}_{6}$ & 0 & 0.6 & 0 & -0.9 & 0 & 0 & 0 & 0 & 0 \\
\hline $\mathrm{C}_{7}$ & 0 & 0 & -0.8 & 0 & 0 & 0.6 & 0 & 0 & 0.5 \\
\hline $\mathrm{C}_{8}$ & 0 & 0 & 0.8 & 0.5 & 0.5 & 0 & 0 & 0 & 0 \\
\hline $\mathrm{C}_{9}$ & 0 & 0.5 & -0.6 & -0.6 & 0 & 0.6 & 0 & 0 & 0 \\
\hline
\end{tabular}


The previous FCM representation and aggregation methods have some limitations in relation to representation and aggregation of perceptions. The first limitation is the lack of an adequate fuzzy method to represent the different kinds of fuzzy connection values of FCMs proposed by different participants. The second limitation is the lack of a comprehensive aggregation method that can aggregate the conflicting and shared perceptions without loss of information, take into account acceptable credibility weights to these perceptions, and deal with fuzzy linguistic and numeric values at the same time.

To overcome these limitations, this paper uses the 2-tuple fuzzy linguistic representation model Herrera and Martinez (2000a) for the representation process. For the aggregation process, this paper proposes a novel FCM fuzzy aggregation method based on this model. The 2-tuple model can represent the connection values of FCM in a fuzzy way throughout the FCM aggregation process. It can also deal with linguistic and numeric values that may be typically encountered during the development of different FCMs. In addition, the proposed fuzzy aggregation method considers the different credibility weights of FCMs to prioritize the importance of connection weights in an FCM before combining with other FCMs. To obtain credibility weights $C W$ for FCMs, this paper utilizes a Consensus Centrality Measure (CCM) of nodes as proposed in Obiedat et al. (2011) to obtain a CCM for the FCMs themselves. This measure is then used to assign credibility weights $C W$ to the FCMs. With these advancements, the proposed FCM aggregation process can approach an accurate consensus perception/FCM without loss of information. To achieve the above, this paper is structured as follows: Section 2 provides an overview of the concept of the 2-tuple fuzzy representation model. Section 3 shows how this model presents FCM data. Section 4 describes the process of obtaining the credibility weights of FCMs. The proposed novel FCM fuzzy aggregation process is presented in Section 5. Finally, Section 6 concludes the paper and suggests some future work.

\section{A 2-TUPLE FUZZY LINGUISTIC REPRESENTATION MODEL}

The main advantage of the 2-tuple model is tackling the limitation of loss of information existing in other classical representation models such as semantic and symbolic models. The information loss implies a lack of precision in the final results and it is caused by linguistic information processing such as the representation and computation processes performed on linguistic values. It offers a useful representation of linguistic values to enhance linguistic processes and frameworks based on linguistic information that could be used in solving various problems Martinez and Herrera (2012). A computational model based on the 2-tuple fuzzy linguistic approach is characterized by accuracy, consistency, and simplicity Herrera and Martinez (2001). Finally, and based on this approach, it is easy to combine linguistic and numeric fuzzy values with different granularity and/or semantic Herrera et al. (2000) Herrera and Martinez (2000b). A 2-tuple fuzzy model was developed based on a symbolic linguistic model Delgado et al. (1993). Here this model is presented first with some definitions followed by an example. Its basis is a linguistic term set $S$ containing $g$ linguistic terms such as low, medium, high etc. and corresponding fuzzy sets (membership functions). For example, consider the FCM in Figure 1, and suppose $S=\left(s_{-6}, s_{-5}, s_{-4}, s_{-3}, s_{-2}, s_{-1}, s_{0}, s_{1}, s_{2}, s_{3}, s_{4}, s_{5}, s_{6}\right)$ and its fuzzy sets that represent FCM weights in $[-1,1]$ universe of discourse are as shown in Figure 3.

Definition 1. Let $S=\left\{s_{0}, \ldots s_{i}, \ldots s_{g}\right\}$ be a linguistic term set and $\beta \in[0, g]$ the result of an aggregation of the labelled indices $i$ in the linguistic term set $S$, i.e., the result of a symbolic aggregation operator. Let $i=\operatorname{round}(\beta)$ and $\alpha=\beta-i$ be two values, such that $i \in[0, g]$ and $\alpha \in[-0.5,0.5)$; then $\alpha$ is called a symbolic translation Herrera and Martinez (2000a).

It can be seen from Definition 1 that $\alpha$ value represents the difference between $\beta$ value resulting from a symbolic aggregation operation and the index value, $i$, of the closest linguistic term $s_{i}$ in $S$. Then, a pair of symbolic values $\left(s_{i}, \alpha_{i}\right), s_{i} \in S$ and $\alpha_{i} \in[-0.5,0.5)$, which represents the means of the 2-tuple linguistic representation model is defined. 
Definition 2. Let $S=\left\{s_{0}, \ldots s_{i}, \ldots s_{g}\right\}$ be a linguistic term set and $\beta \in[0, g]$ represents the result of linguistic symbolic aggregation, then the 2-tuple $\left(s_{i}, \alpha\right)$ equivalent of $\beta$ denoted as $\Delta(\beta)$ is obtained with the following function Herrera and Martinez (2000a):

$$
\Delta(\beta)=\left\{\begin{aligned}
s_{i}, & i=\text { round }(\beta) \\
\alpha=\beta-i, & \alpha \in[-0.5,0.5)
\end{aligned}\right.
$$

where round (.) is the usual round operation, $S_{i}$ has the closest index label to $\beta$ and $\alpha$ is the value of the symbolic translation.

Definition 3. Let $S=\left\{s_{0}, \ldots s_{i}, \ldots s_{g}\right\}$ be a linguistic term set and $\left(s_{i}, \alpha\right)$ be a 2-tuple, then the equivalent numerical value $\beta$ to 2 -tuple $\left(s_{i}, \alpha\right)$ denoted as $\Delta^{-1}\left(s_{i}, \alpha_{i}\right)$ is obtained with the following function:

$\Delta^{-1}\left(s_{i}, \alpha\right)=i+\alpha=\beta$

Definition 4. Let $s_{i} \in S$ be a linguistic term, then its equivalent 2-tuple representation is obtained by adding a value 0 as a symbolic translation Herrera and Martinez (2000a):

$\Delta\left(s_{i}\right)=\left(s_{i}, 0\right)$

Example 1. Let $S=\left\{s_{0}, \ldots s_{i}, \ldots s_{g}\right\}$ be a linguistic term set where each linguistic term $S_{i}$ is represented by real valued triangular parameters $\left(a_{i}, b_{i}, c_{i}\right)$ and the membership function of a value $(n)$ associated with $S_{i}$ $\left(\mu_{s_{i}}(n)\right)$ is calculated using the following Equation Obiedat et al. (2011):

$\mu_{s_{i}}(n)=\left\{\begin{array}{cl}\frac{n-a_{i}}{b_{i}-a_{i}}, & a \leq n \leq b \\ \frac{c_{i}-n}{c_{i}-b_{i}}, & b<n \leq c \\ 0, & \text { otherwise }\end{array}\right.$

Then $\beta$, the result of the symbolic aggregation process over labels assessed in $S$ is obtained using the following Equation Obiedat et al. (2011):

$$
\beta=\frac{\sum_{i=0}^{g} i \cdot \mu_{s_{i}}}{\sum_{i=0}^{g} \mu_{s_{i}}}
$$

Let $\beta=2.6$, then $\Delta(\beta)=\left(s_{i}, \alpha\right)$ or the representation of $\beta=2.6$ by means of a 2 -tuple which consists of values $(i, \alpha)$ where $i=$ round $(2.6)=3$ and the symbolic translation $\alpha=\beta-i$ or $\alpha=2.6-3=-0.4$, becomes $\Delta(2.6)=\left(s_{3},-0.4\right)$ (Figure 2$)$.

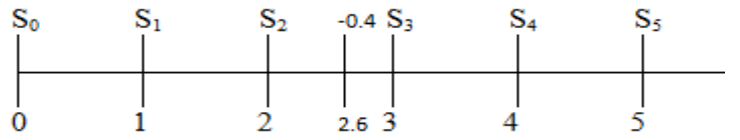

$\left(\mathrm{S}_{3},-0.4\right)$

Figure 2. Representation of $\beta=2.6$ and its 2tuple representation involving the closest linguistic term $S_{3}$ and the symbolic translation $\alpha=-0.4$

\section{FCM DATA - FUZZY REPRESENTATION}

The proposed FCM aggregation method first uses the 2-tuple model to represent the FCM connection weight values in terms of $\beta$. The representation method starts by determining the linguistic set $S$ and its fuzzy sets (membership functions) that represent each FCM weight value as shown in Figure 3. Based on this linguistic 
set, it uses Equation 4 to calculate the membership function values of FCM weights to convert them into fuzzy sets assessed in $S$. Finally, it transforms these fuzzy sets into $\beta$ values using Eq. 5.

For the FCM in Figure 1 with connection values given in Table 1, its corresponding linguistic term set $\mathrm{S}$ with fuzzy sets shown in Figure 3 produces $\beta$ values given in Table 2 .

\section{CREDIBILITY WEIGHTS}

This section describes how credibility weights $C W$ of FCMs are obtained based on their CCM values. Initially, a brief description of CCM definition and calculation is recalled from Obiedat et al. (2011). The CCM is obtained using 3 common centrality measures: Degree, Closeness and Betweenness. The degree centrality measure $\operatorname{Cen}_{D}\left(c_{i}\right)$ represents the centrality of node $c_{i}$ according to its directed connections. It measures the strength of the direct connections of node $c_{i}$ with other nodes in the FCM and is calculated by the sum of the absolute connection weights entering node $c_{i}$, this measure is called indegree $\left(i d\left(c_{i}\right)\right.$, and exiting node $c_{i}$, this measure is called outdegree $\left(\operatorname{od}\left(c_{i}\right)\right.$ :

$\operatorname{Cen}_{D}\left(c_{i}\right)=\sum\left(i d\left(c_{i}\right)+o d\left(c_{i}\right)\right)$

The Closeness centrality measure $\operatorname{Cen}_{C}\left(c_{i}\right)$ represents the centrality of node $c_{i}$ based on the shortest paths between node $c_{i}$ and all other nodes in FCM. It measures how close node $c_{i}$ is to other nodes:

$$
\operatorname{Cen}{ }_{C}\left(c_{i}\right)=\frac{1}{\sum_{t=1}^{N} d_{G}\left(c_{i}, t\right)}
$$

where $t \neq c_{i}, N$ is the number of nodes, and $d_{G}\left(c_{i}, t\right)$ is the shortest path between nodes $c_{i}$ and $t$. Finally, like closeness, the betweenness centrality measure $\operatorname{Cen}_{B}\left(c_{i}\right)$ depends on the shortest paths between 
node $c_{i}$ and all other nodes in FCM to calculate the centrality of node $c_{i}$. It measures how much node $c_{i}$ controls communication between other nodes and spreads information through the FCM:

$\operatorname{Cen}_{B}\left(c_{i}\right)=\sum_{s, t=1}^{N} \frac{\sigma_{s t}\left(c_{i}\right)}{\sigma_{s t}}$

where $s \neq t \neq c_{i}, \sigma_{s t}$ is the number of shortest paths from node $s$ to node $t$, and $\sigma_{s t}\left(c_{i}\right)$ is the number of shortest paths from $s$ to $t$ that passes through node $c_{i}$.

Now, the above measures of nodes are used to calculate the degree, closeness and betweenness for the FCMs themselves. Let $\mathrm{Cen}_{D}^{*}, \mathrm{Cen}_{C}^{*}$ and $\mathrm{Cen}_{B}^{*}$ are the maximum degree, closeness and betweenness centrality values of nodes in FCM respectively. Then, the degree, closeness and betweenness for the FCM are calculated by the following Equations 9, 10 and 11, respectively:

$$
\begin{aligned}
& \operatorname{Cen}_{D}(F C M)=\frac{\sum_{i=1}^{N}\left(\operatorname{Cen}_{D}^{*}-\operatorname{Cen}_{D}\left(c_{i}\right)\right)}{N-1} \\
& \operatorname{Cen}_{C}(F C M)=\frac{\sum_{i=1}^{N}\left(\operatorname{Cen}_{C}^{*}-\operatorname{Cen}_{C}\left(c_{i}\right)\right)}{(N-1)(N-2) /(N-3)} \\
& \operatorname{Cen}_{B}(F C M)=\frac{\sum_{i=1}^{N}\left(\operatorname{Cen}_{B}^{*}-\operatorname{Cen}_{B}\left(c_{i}\right)\right)}{N-1}
\end{aligned}
$$

These measures are then prioritized according to their importance and effectiveness in determining FCM centrality. This is done subjectively by the developer of the system by assigning non-negative prioritisation weights $b_{D}, b_{C}$ and $b_{B}$ for the 3 measures of the degree, closeness and betweenness, respectively, where $b_{D}+b_{C}+b_{B}=1$. Finally, the CCM of the FCM, $\operatorname{Cen}_{\text {Cons }}(F C M)$, is calculated as follows:

$\operatorname{Cen}_{\text {Cons }}(F C M)=b_{D} \bullet \operatorname{Cen}_{D}(F C M)+b_{C} \bullet C e n_{C}(F C M)+b_{B} \bullet \operatorname{Cen}_{B}(F C M)$

These calculations are repeated to obtain CCM for all FCMs in the system. These CCMs are normalized in the range $[0,1]$ and summed. Finally, the credibility weight of each FCM, $c w_{F C M}$, is calculated by dividing its normalized CCM by the normalised sum of CCMs.

\section{FCM FUZZY AGGREGATION PROCESS}

Once the credibility weights of FCMs are defined and their connection weights are represented in $\beta$ values, the first step of the proposed FCM aggregation process is to initialize an adjacency matrix ( $G r p_{F C M}$ ) of zero values for the group of FCMs as follows:

$$
\operatorname{Grp}_{F C M_{i j}}=\{0 \quad i, j=1 \cdots N C\}
$$

where $\operatorname{Grp}_{F C M_{i j}}$ represents the connection weight $(\beta)$ between node $c_{i}$ and node $c_{j}$ in the group FCM, and $N C$ is the number of different nodes in all FCMs in the group. 
Then, for each FCM in the group $\left(F C M_{k}\right)$, its matrix is augmented to include the different nodes in all FCMs. Each column and row of any new node added to an FCM matrix are filled with zero values. Then, the connection values $(\beta)$ of $F C M_{k}$ are weighted by its credibility weight $\left(c w_{k}\right)$ as follows:

$F C M_{w k}=F C M_{k} \bullet c w_{k}$

where $F C M_{w k}$ is the weighted connection matrix of $F C M_{k}$.

The next step is to combine the $F C M_{w k}$ to the $\operatorname{Grp}_{F C M}$ as follows:

$\operatorname{Grp}_{F C M}=\operatorname{Grp}_{F C M}+F C M_{w k}$

Finally, these steps of FCM aggregation process are repeated until all the FCM matrices in the group are aggregated to $G r p_{F C M}$. The $G r p_{F C M}$ is the social group FCM that represents the consensus perception. The Group FCM now can be used to analyse Group systems dynamics.

\section{CONCLUSION AND FUTURE WORK}

This article utilizes the 2-tuple fuzzy representation model and CCM measure to propose new fuzzy methods for FCM representation and aggregation. It benefits from the 2-tuple model in the representation of the connection values of FCMs in fuzzy $\beta$ values. It also uses these fuzzy $\beta$ values in the aggregation process. In addition, it also utilizes the CCM of FCMs to assign credibility weights to these FCMs in order to properly account for different levels of knowledge of FCM developers. As a result, the proposed FCM aggregation process uses robust calculations to achieve accurate and proper consensus group perception/FCM. In future work, we plan to propose a new semi-quantitative method to condense a large FCM into a small, easily understandable and traceable one.

\section{REFERENCES}

Delgado, M., Verdegay, J. L., \& Vila, M. A. (1993). On aggregation operations of linguistic labels. International Journal of Intelligent Systems, 8(3), 351-370.

Dickerson, J. A., \& Kosko, B. (1994). Virtual Worlds as Fuzzy Cognitive Maps. Presence, 3(2), 73-89.

Herrera, F., Herrera-Viedma, E., \& Martínez, L. (2000). A fusion approach for managing multi-granularity linguistic term sets in decision making. Fuzzy Sets and Systems, 114(1), 43-58.

Herrera, F., \& Martinez, L. (2000a). A 2-tuple fuzzy linguistic representation model for computing with words [Article]. Ieee Transactions on Fuzzy Systems, 8(6), 746-752.

Herrera, F., \& Martinez, L. (2000b). An approach for combining linguistic and numerical information based on the 2-tuple fuzzy linguistic representation model in decision-making [Article]. International Journal of Uncertainty Fuzziness and Knowledge-Based Systems, 8(5), 539-562.

Herrera, F., \& Martinez, L. (2001). The 2-Tuple Linguistic Computational Model: Advantages of its Linguistic Description, Accuracy and Consistency. International Journal of Uncertainty, Fuzziness and Knowledge-Based Systems, 09(supp01), 33-48.

Kosko, B. (1986). Fuzzy cognitive maps [Article]. International Journal of Man-Machine Studies, 24(1), 6575.

Martinez, L., \& Herrera, F. (2012). An overview on the 2-tuple linguistic model for computing with words in decision making: Extensions, applications and challenges. Information Sciences, 207(0), 1-18.

Obiedat, M., Samarasinghe, S., \& Strickert, G. (2011). A New Method for Identifying the Central Nodes in Fuzzy Cognitive Maps using Consensus Centrality Measure. Paper presented at the meeting of the 19th International Congress on Modelling and Simulation, Perth, Australia.

Ozesmi, U., \& Ozesmi, S. (2003). A participatory approach to ecosystem conservation: Fuzzy cognitive maps and stakeholder group analysis in Uluabat Lake, Turkey [Article]. Environmental Management, 31(4), 518-531.

Ozesmi, U., \& Ozesmi, S. (2004). Ecological models based on people's knowledge: a multi-step fuzzy cognitive mapping approach [Article]. Ecological Modelling, 176(1-2), 43-64.

Strickert, G., Smarasinghe, S., Doscher, C., \& Davies, T. (2010). A Gap Hazard Analysis: Initiating Policy Development with Mountainous Communities. Journal of Natural Resources Policy Research, 2(4), 389-407. 